American Journal of Animal and Veterinary Sciences 3 (1): 40-42, 2008

ISSN 1557-4555

(C) 2008 Science Publications

\title{
Survey on Seroprevalence of Anti-Toxoplasma Gondii Antibodies in Cattle in Tabriz (Iran) by IFAT
}

\author{
${ }^{1}$ A. Nematollahi and ${ }^{2}$ Gh. Moghddam \\ ${ }^{1}$ Department of Pathobiology, Faculty of Veterinary Medicine, \\ University of Tabriz, Tabriz, Iran \\ ${ }^{2}$ Department of Clinical Science, Faculty of Veterinary Medicine, \\ University of Tabriz, Tabriz, Iran
}

\begin{abstract}
Toxoplasmosis is a zoonotic disease in various species of animals caused by a sporozoan parasite, Toxoplasma gondii. The disease occurs throughout the world including Iran. This disease is a common infection of sheep, goats and it is recognized as one of major infection cause of reproductive failure. This survey was carried out to show the prevalence of this disease in cattle in Tabriz (Iran). 490 sera samples were collected from both native and industrial cattle. The Indirect Fluorescent Antibody Test (IFAT) was used to determine the rate of infection. The results of this survey are showed that the overall seroprevalence of antibody of Toxoplasma incattle in Tabriz is $15.91 \%$. There was significant differences in infection to Toxoplasma gondii in the age group of cattle $(\mathrm{P}<0.05)$. The prevalence of anti-toxoplasma antibody in cattle in the $<1$ years old was higher than $>1$ years old cattle. Also a significant differences was observed between rate of infection and sex in cattle $(\mathrm{P}<0.01)$. In total the results of this study together with the previously recorded low seroprevalence of antibody to Toxoplasma in cattle in Iran and other countries support the impression that Toxoplasmosis is not widespread cause for abortion and a latent infection in cattle.
\end{abstract}

Key words: Seroprevalence, toxoplasma, cattle, Tabriz, Iran

\section{INTRODUCTION}

Toxoplasmosis is a zoonotic disease in various species of animals caused by a sporozoan parasite, Toxoplasma gondii, distributed throughout the world including Iran. Toxoplasma gondii is a heteroxenous coccidian parasite ${ }^{[2]}$. The definitive hosts are domestic cats and various species of wild felids. The intermediate hosts are mammals and birds. A consideration of its transmission route provides an indication of why it may be predicted to alter host behaviour. T. gondii has an indirect life cycle, where members of the cat family (Felidae) are the only known definitive hosts able to shed oocysts with their faeces.

These oocysts contaminate the surrounding environment ${ }^{[11]}$. A 20 gr cat stool can contain between 2-20 million oocysts and after faecal decomposition, the local soil contamination can be as high as 1, 00,000 oocysts $\mathrm{gr}^{-1}$. They remain infectious for more than 1 year ${ }^{[6]}$. If oocysts are ingested by another cat viable organisms are released which invade intestinal epithelial cells, where they undergo both asexual and sexual cycle. In contrast, if oocysts are ingested by a non-feline host, such as a wild rodent, bird or human, an extra-intestinal cycle is observed, where asexual reproduction occurs and small thin-walled cysts form in all nucleated cells, particularly those of the brain. If a native cat consumes infected intermediate host prey, the T. gondii life cycle is completed ${ }^{[7]}$. The infection has been found in many zoogeographic regions and high reactor rates were found among domestic animals. Upon ingestion by a nonfeline, secondary host such as a rodent, bird, or human, the sporozoite, unable to complete the reproductive portion of its life cycle, enters the asexual cycle, which leads to the formation of trophozoites. Parasitemia follows, at which point $T$. gondii can cross the placenta and infect the fetus. ${ }^{[2]}$ An immune response is initiated, and the infected organism then acquires lifelong immunity. The acute asexual phase is characterized by the tachyzoite. This form of the organism is very sensitive to extreme temperatures, is highly infective, and can penetrate the buccal mucosa. The tachyzoite can invade almost any cell and does so very quickly using specialized organelles for penetration. It forms a pseudocyst of host and parasite origin that prevents destruction by host lysosomes. The

Corresponding Author: Ahmad Nematollahi, Department of Parasitology, Faculty of Veterinary Medicine,

University of Tabriz, Tabriz, Iran 
tachyzoite multiplies rapidly in this protected environment until the host cell ruptures and further dissemination can occur. Because of the lack of specific clinical manifestations during acute infection, T. gondii is mainly a laboratory diagnosis. Definitive diagnosis is important in pregnant patients because they will benefit most from early treatment or prophylaxis against the sequelae of acute toxoplasmosis.The diagnosis of $\mathrm{T}$. gondii abortion is based on the detection of specific antibodies in the adult population by Indirect Fluorescent Antibody Test (IFAT) or Latex Agglutination Test (LAT) and the visualization of characteristic histologic lesions in the placenta and the brain of the aborted fetus ${ }^{[8]}$.

The purpose of this study was to determine the prevalence of anti-Toxoplasma antibodies in cattle of Tabriz (Iran). The Indirect Flourecent Antibody Test (IFAT) was used to determine the rate of infection ${ }^{[5]}$.

\section{MATERIALS AND METHODS}

A total 490 serum samples were taken from adult cattle (263 female and 127 male) that were in different area of husbandry farms in Tabriz. The cattle were divided in two age groups, $<1$ years old and $<1$ years old. Blood was collected from cattle by vein puncture of the jugular vein. Sera were harvested following centrifugation of clotted blood, which was stored at $20^{\circ} \mathrm{C}$ until needed. Antibodies to T. gondii were tested by Indirect Fluorescent Antibody Test (IFAT). Antigen of T. gondii was provided by Pasteur Institute of Iran (Rh strain). Antibovine congucated were purchased Hakimi Co and is diluted 1:20. Firstly, sera were diluted 1:20, 1:100 and 1:400 in phosphate-buffered saline (PBS, 0.1 M phosphate, 0.33 M NaCI) $(\mathrm{pH}=7.2)$. Aliquot of $10 \mu \mathrm{L}$ from each serum was placed on the well of $\mathrm{T}$. gondii-slide and incubated in a humidified chamber at $37^{\circ} \mathrm{C}$ for $30 \mathrm{~min}$. After washing in PBS, slides were incubated for $30 \mathrm{~min}$ at $37^{\circ} \mathrm{C}$. Slides were washed three times in PBS, counterstained with $1 \%$ Evans Blue and examined for fluorescein in a light microscope. Positive and negative bovine control sera were included in each slide. Finally $\mathrm{X}^{2}$-test was used determine any statistical association between the seroprevalence of Toxoplasmosis in cattle and sex and age.

\section{RESULTS AND DISCUSSION}

Until now insufficient data are available on cattle toxoplasmosis in the world, and there has been no
Table 1: The seroprevalence of anti-Toxoplasma gondii antibodies in cattle in Tabriz

\begin{tabular}{lll}
\hline No. tested & No. positive & \% positive \\
\hline 490 & 78 & 15.91 \\
\hline
\end{tabular}

Table 2: The seroprevalence of anti-Toxoplasma gondii antibodies in cattle by age in Tabriz

\begin{tabular}{llll}
\hline Age group & No. tested & No. positive & $\%$ positive \\
\hline$<1$ year & 302 & 82 & 43.61 \\
$>1$ year & 188 & 29 & 9.6 \\
\hline
\end{tabular}

Table 3: The seroprevalence of anti-Toxoplasma gondii antibodies in cattle by sex in Tabriz

\begin{tabular}{llll}
\hline Sex & No. tested & No. positive & \% positive \\
\hline Male & 127 & 36 & 28.34 \\
Female & 263 & 32 & 12.16 \\
\hline
\end{tabular}

report on cattle toxoplasmosis from Iran. In total 490 cattle sera were tested by IFA test. Positive 1:20 and more were found in $78(15.91 \%)$ cattle

(Table 1). The prevalence of anti-toxoplasma antibody in cattle by age is shown in Table 2 . The prevalence in cattle in the $<1$ years old was higher than $>1$ years old cattle. This difference was statistically significant $(\mathrm{P}<0.05)$ (Table 2).

Totally $36(28.34 \%)$ of 127 male cattle and $32(12.16 \%)$ of 263 female cattle were seropositive for T. gondii (Table 3). This difference was statistically significant $(\mathrm{P}<0.01)$.

Most epidemiological investigations on Toxoplasmosis in Iran were based on the results of serological examination by Sabin-Feldman dyetest (DT) or Latex Agglutination Test (LAT) and most of them were on the human and ovine infection. Therefore insufficient data are available on Toxoplasmosis on cattle in Iran. The IFA test is a relatively simple assay for assessing the infection status of animals and is especially useful for screening large number of samples ${ }^{[8]}$. The results of this survey are showed that the overall seroprevalence of cattle to antibody of Toxoplasma in Tabriz is $15.91 \%$. Similar results (16.21\%) were found by Hoghoogi-rad et al. (1993) in Ahwaz, southwest Iran on cattle By IFA test ${ }^{[4]}$.

In other study on seroprevalence of antitoxoplasma gondii antibody in buffaloes in Khoozestan province in Iran, that was tested by IFA test, the rate was reported $8.8 \%{ }^{[9]}$. Differencess are probably due to this cause that buffaloes may not act as a suitable host for Toxoplasma. Hashemi-Fesharaki (1996) tested sera from 2000 cows by Latex Agglutination Test (LAT) and found no positive reactions in cow sera ${ }^{[3]}$. This different are probably due to different method for testing and lower degree of feline presence in location 
of his study. In general seropositivity to Toxoplasma in cattle is not high and in studies by LAT on Toxoplasma in other countries also the rate of infection was low. This rate was recorded in three studies1.03, 5.5 and $16.10 \%$ in Brazil, Vietnam and Bangladesh, respectively ${ }^{[5,10,12]}$.

The results of this study also is showed that cattle under the age of one year were significantly $(\mathrm{P}>0.05)$ more infected the older cattle. This result confirm the experimental findings of some workers such as Deuby and Beattie (1988) who used calves as the suitable models for any detailed research on Toxoplasmosis. It seems that the cattle deplet Toxoplasma antibodies as it age increase ${ }^{[1]}$.

The results of this study revealed that antibody to Toxoplasma in male cattle were significantly more than females. This finding is similar to Samad et al., finding in Bangladesh ${ }^{[12]}$ and is adversed to research of Pita $e t$ al., research in Brazil (1993) who reported females cattle was more seropositive ${ }^{[10]}$. This difference are probably due to different management methods in breeding of animals.

In total the results of this study together with the previously recorded low seroprevalence in cattle in Iran and other countries support the impression that Toxoplasmosis is not widespread cause for abortion and a latent infection in cattle. Furthermore cattle are not suitable host for Toxoplasma gondii.

\section{ACKNOWLEDGEMENTS}

This research was founded by cooperation of Dr.Ardakanian and Dr.Naghshineh. We are grateful for their assistance in collection of serum samples.

\section{REFERENCES}

1. Deuby, J.P. and C.P. Beattie, 1988. Toxoplasmosis of Animals and Man. CRC Press, Boca Raton, USA, pp: 200-02.

2. Gagne, S.S., 2001. Toxoplasmosis, Primary Care Update for OB Gyns $^{-1}$, 18: 122-126.

3. Hashemi-Fesharaki, R., 1994. A serological survey on toxoplasmosis in cattle, sheep and goats in Iran. Archive of Institute Razi, 44: 56-64.
4. Hoghoogi-Rad, N., M. Razi-Jalali, A.R. SadreBazzaz, M. Seadat-Amolii, S. Ataii kachooii and A. Bozorgnia, 1995. Toxoplasmosis in cats, sheep, cattle and some birds in Ahwaz area. center of Khoozestan province, Iran: Abstracts. XXV Congress of the World Veterinary Association, Yokohama, Japan.

5. Huong, L.T., B.L. Ljungstrom, A. Uggla and B. Bjorkman, 1998. Prevalence of antibodies to Neospora caninum and Toxoplasma gondii in cattle and water buffaloes in southern Vietnam. Vet. Erinary Paras., 75: 53-57.

6. Hutchison, J.F., J. Dunachie Siim and K. Work, 1996. The life cycle of Toxoplasma gondii. Brazil Med. J., 53: 806.

7. Levine, N.D., 1985. Veterinary Protozoloology. Lowa state University Press, USA, pp: 248-59.

8. Markell, D., T. John and W.A. Krotoski, 1999. Markell and Vogue's Medical Parasitoloty, W.B. Saunders Company, Philadelphia, USA, pp: 347-58.

9. Navidpour, S.H. and N. Hoghoogi-Rad, 1998. Seroprevalence of anti-Toxoplasma gondii antibodies in buffaloes in Khoozestan province, Iran. Vet. Pars., 77: 191-94.

10. Pita Gondim, L., F. Barbosa Jr., H.V.C. Ribeiro Filho and H. Saek, 1999. Serological survey of antibodies to Toxoplasma gondii in goats, sheep, cattle and water buffaloes in Bahia State, Brazil. Vet. Paras., 82: 273-76.

11. Roberts, J. and J. Janovy, 2000. Schmidt and Roberts Foundations of Parasitology, McGraw-Hill Companies, Boston, USA, pp: 546-64.

12. Samad, M.A., K.B. Rahman and A.K. Halder, 1993. Seroprevalence of Toxoplasma gondii in domestic ruminants in Bangladesh. Vet. Paras., 47: $157-59$. 